\title{
Perforation Bile Duct, CTCAE
}

National Cancer Institute

\section{Source}

National Cancer Institute. Perforation Bile Duct, CT CAE. NCI Thesaurus. Code C143742.

A disorder characterized by a rupture in the wall of the extrahepatic or intrahepatic bile duct. 